\title{
CONVEX MATRIX FUNCTIONS
}

\author{
WILLIAM WATKINS
}

\begin{abstract}
The purpose of this paper is to prove convexity properties for the tensor product, determinant, and permanent of hermitian matrices.
\end{abstract}

Let $C^{n}$ be the vector space of all complex $n$-tuples with the usual inner product ( , ) and let $H_{n}$ be the set of all $n$ by $n$ hermitian matrices. A matrix $A$ in $H_{n}$ is nonnegative if $(A x, x) \geqq 0$ for all $x$ in $C^{n}$. If $A$ and $B$ are in $H_{n}$, we write $A \geqq B$ if $A-B$ is nonnegative. A function $f$ from $H_{n}$ to $H_{m}$ is monotone if $A \geqq B$ implies $f(A) \geqq f(B)$, and convex if $f(\lambda A+(1-\lambda) B) \leqq \lambda f(A)+(1-\lambda) f(B)$, for all $0 \leqq \lambda \leqq 1$.

Löwner [6] introduced the case where $f$ is induced by a real valued function and $m=n$. Other authors [2], [4], [5] have analysed this case further.

EXAMPLE [9]. The inverse function is convex on the set of all invertible, nonnegative matrices in $H_{n}$.

EXAMPLE [4]. The square root function is monotone on the set of all nonnegative matrices in $H_{n}$.

Some work has been done on the case where $m=1$. That is, $f$ is a function from $H_{n}$ to the real numbers. For example, Marcus and Nikolai [8] have shown that each member of a class of generalized matrix functions is monotone. This class of functions contains the determinant and permanent. For other results of this type see [1].

In order to state the convexity property for the tensor product, let $m_{1}, \cdots, m_{r}$ be $r$ positive integers. It is well known [10, p. 268] that, for $x_{i}, y_{i}$ in $C^{m_{i}}, i=1, \cdots, r$, the decomposable tensors $x_{1} \otimes \cdots \otimes x_{r}$ and $y_{1} \otimes \cdots \otimes y_{r}$ in $C^{N}, N=m_{1} \cdots m_{r}$, satisfy

$$
\left(x_{1} \otimes \cdots \otimes x_{r}, y_{1} \otimes \cdots \otimes y_{r}\right)=\left(x_{1}, y_{1}\right) \cdots\left(x_{r}, y_{r}\right) .
$$

If $A_{i}$ is an $m_{i}$ by $m_{i}$ matrix $(i=1, \cdots, r)$, then the tensor product $\otimes^{r} A_{i}$ is an $N$ by $N$ matrix satisfying

$$
\otimes^{r} A_{i}\left(x_{1} \otimes \cdots \otimes x_{r}\right)=A_{1} x_{1} \otimes \cdots \otimes A_{r} x_{r},
$$

for $x_{i}$ in $C^{m_{i}}(i=1, \cdots, r)$.

Received by the editors June 15, 1973.

AMS (MOS) subject classifications (1970). Primary 15A45; Secondary 15A15, $15 \mathrm{~A} 69$. 
THEOREM 1. If $A_{i}$ and $B_{i}$ are matrices in $H_{m_{i}}$ with $0 \leqq B_{i} \leqq A_{i}, i=1, \cdots$, $r$, and $0 \leqq \lambda \leqq 1$, then

$$
\otimes^{r}\left(\lambda A_{i}+(1-\lambda) B_{i}\right) \leqq \lambda \otimes^{r} A_{i}+(1-\lambda) \otimes^{r} B_{i} .
$$

Definition (Generalized matrix function). Let $S_{n}$ denote the permutation group on $n$ letters and let $G$ be a subgroup of $S_{n}$ with irreducible character $\chi: G \rightarrow C$. For each $n$ by $n$ complex matrix $A=\left(a_{i j}\right)$, define

$$
d(A)=\sum \chi(\sigma) \prod_{i=1}^{n} a_{\sigma i, i} \quad(\operatorname{sum} \sigma \text { in } G) .
$$

The function $d$ depends on both the subgroup $G$ and its character $\chi$. If $G=S_{n}$ and $\chi(\sigma)$ is the sign of $\sigma$, then $d$ is the determinant function. If $G=S_{n}$ and $\chi \equiv 1$, then $d$ is the permanent function. For a fuller explanation see [7].

THEOREM 2. If $A$ and $B$ are matrices in $H_{n}$ with $0 \leqq B \leqq A$ and $0 \leqq \lambda \leqq 1$, then

$$
d(\lambda A+(1-\lambda) B) \leqq \lambda d(A)+(1-\lambda) d(B) .
$$

Corollary. If $A$ and $B$ are matrices in $H_{n}$ with $0 \leqq B \leqq A$ and $0 \leqq \lambda \leqq 1$, then

and

$$
\operatorname{det}(\lambda A+(1-\lambda) B) \leqq \lambda \operatorname{det} A+(1-\lambda) \operatorname{det} B
$$

$$
\operatorname{per}(\lambda A+(1-\lambda) B) \leqq \lambda \operatorname{per} A+(1-\lambda) \operatorname{per} B .
$$

Proofs.

Proof of THEOREM 1. It is shown in [8] that if $A_{1}, B_{1}$ are in $H_{m_{1}}$ and $A_{2}, B_{2}$ are in $H_{m_{2}}$ with $0 \leqq B_{1} \leqq A_{1}$ and $0 \leqq B_{2} \leqq A_{2}$, then $A_{1} \otimes A_{2} \geqq$ $B_{1} \otimes B_{2}$. Thus the right side of the identity

$$
\begin{aligned}
\lambda\left(A_{1} \otimes A_{2}\right) & +(1-\lambda)\left(B_{1} \otimes B_{2}\right)-\left(\lambda A_{1}+(1-\lambda) B_{1}\right) \\
& \otimes\left(\lambda A_{2}+(1-\lambda) B_{2}\right)=\lambda(1-\lambda)\left(A_{1}-B_{1}\right) \otimes\left(A_{2}-B_{2}\right)
\end{aligned}
$$

is nonnegative. Theorem 1 follows by induction.

In order to prove Theorem 2, we develop ideas relating the tensor product to the generalized matrix function $d$.

For each $\sigma$ in $S_{n}$, define an $N$ by $N\left(N=n^{n}\right)$ permutation matrix $P(\sigma)$ by $P\left(\sigma^{-1}\right) x_{1} \otimes \cdots \otimes x_{n}=x_{\sigma 1} \otimes \cdots \otimes x_{\sigma n}$ for all $x_{i}$ in $C^{n}$. Notice that $P(\sigma \mu)=P(\sigma) P(\mu)$. Define an $N$ by $N$ matrix $T$ by

$$
T=\frac{\chi(1)}{|G|} \sum \chi(\sigma) P(\sigma) \quad(\operatorname{sum} \sigma \text { in } G) .
$$


It follows from the orthogonality relations for irreducible characters [3, p. 219] that $T$ is an idempotent. The matrix $T$ is hermitian since the complex conjugate of $\chi(\sigma)$ is $\chi\left(\sigma^{-1}\right)$ and $P(\sigma)^{*}=P\left(\sigma^{-1}\right)$. If $A=\left(a_{i j}\right)$ is an $n$ by $n$ matrix, then $\otimes^{n} A$ commutes with each $P(\sigma)$ and so it commutes with $T$.

Let $e_{1}, \cdots, e_{n}$ be the usual basis for $C^{n}$. Then,

$$
\begin{aligned}
\left(\left(\otimes^{n} A\right) T e_{1} \otimes \cdots \otimes e_{n},\right. & \left.T e_{1} \otimes \cdots \otimes e_{n}\right) \\
= & \left(T^{*}\left(\otimes^{n} A\right) T e_{1} \otimes \cdots \otimes e_{n}, e_{1} \otimes \cdots \otimes e_{n}\right) \\
= & \left(T\left(\otimes^{n} A\right) e_{1} \otimes \cdots \otimes e_{n}, e_{1} \otimes \cdots \otimes e_{n}\right) \\
= & \left(T A e_{1} \otimes \cdots \otimes A e_{n}, e_{1} \otimes \cdots \otimes e_{n}\right) \\
= & \frac{\chi(1)}{|G|} \sum_{\sigma} \chi(\sigma)\left(A e_{\sigma 1} \otimes \cdots \otimes A e_{\sigma n}, e_{1} \otimes \cdots \otimes e_{n}\right) \\
= & \frac{\chi(1)}{|G|} \sum_{\sigma} \chi(\sigma) \prod_{i}\left(A e_{\sigma i}, e_{i}\right) \\
= & \frac{\chi(1)}{|G|} d(A) .
\end{aligned}
$$

In the second inequality, notice that $T^{*}\left(\otimes^{n} A\right) T=T\left(\otimes^{n} A\right)$, since $T$ and $\otimes^{n} A$ commute and $T$ is a hermitian idempotent. If $A$ and $B$ are in $H_{n}$ and $0 \leqq A \leqq B$ and $0 \leqq \lambda \leqq 1$, then by Theorem 1 we have

$$
\otimes^{n}(\lambda A+(1-\lambda) B) \leqq \lambda \otimes^{n} A+(1-\lambda) \otimes^{n} B .
$$

By comparing inner products

$$
\left(\otimes^{n}(\lambda A+(1-\lambda) B) T e_{1} \otimes \cdots \otimes e_{n}, T e_{1} \otimes \cdots \otimes e_{n}\right)
$$

and

$$
\left(\left(\lambda \otimes^{n} A+(1-\lambda) \otimes^{n} B\right) T e_{1} \otimes \cdots \otimes e_{n}, T e_{1} \otimes \cdots \otimes e_{n}\right),
$$

we get $d(\lambda A+(1-\lambda) B) \leqq \lambda d(A)+(1-\lambda) d(B)$. The corollary consists of special cases.

\section{REFERENCES}

1. R. Bellman, Introduction to matrix analysis, McGraw-Hill, New York, 1960, Chap. 8. MR 23 \#A153.

2. J. Bendat and S. Sherman, Monotone and convex operator functions, Trans. Amer. Math. Soc. 79 (1955), 58-71. MR 18, 588.

3. C. W. Curtis and I. Reiner, Representation theory of finite groups and associative algebras, Pure and Appl. Math., vol. 11, Interscience, New York, 1962. MR 26 \#2519.

4. C. Davis, Notions generalizing convexity for functions defined on spaces of matrices, Proc. Sympos. Pure Math., vol. 7, Amer. Math. Soc., Providence, R.I., 1963, pp. 187201. MR 27 \#5771. 
5. F. Kraus, Über konvexe Matrixfunktionen, Math. Z. 41 (1936), 18-42.

6. K. Löwner, Über monotone Matrixfunktionen, Math. Z. 38 (1934), 177-216.

7. M. Marcus and H. Minc, Generalized matrix functions, Trans. Amer. Math. Soc. 116 (1965), 316-329. MR 33 \#2655.

8. M. Marcus and P. J. Nikolai, Inequalities for some monotone matrix functions, Canad. J. Math. 21 (1969), 485-494. MR 38 \#5815.

9. M. H. Moore, A convex matrix function, Amer. Math. Monthly 80 (1973), 408409.

10. G. D. Mostow and J. H. Sampson, Linear algebra, McGraw-Hill, New York, 1969. MR 42 \#7673.

Department of Mathematics, California State University, Northridge, CALIFORNIA 91324 